\section{Assessing young people who deliberately harm themselves}

\author{
JANE HURRY and PAMELA STOREY
}

\begin{abstract}
Background Many young people who harm themselves have chronic mental health or social problems or are at risk of future self-harm or even suicide. The accident and emergency (A\&E) clinic is an important gateway to treatment.
\end{abstract}

\begin{abstract}
Aims To describe the psychosocial assessment of 12- to 24-year-old patients attending A\&E clinics following deliberate self-harm (DSH) and to identify features of service management and provision which maximise specialist assessment.
\end{abstract}

\begin{abstract}
Method A postal questionnaire was sent to a sample of one in three $A \& E$ departments in England. In a representative sample of 18 of these hospitals, staff were interviewed and 50 case notes per hospital were examined.
\end{abstract}

\section{Results Psychosocial assessment by non-specialist doctors in A\&E departments tended to be of variable quality, focused on short-term risk. Around 43\% of patients aged 12-24 were assessed by a specialist; specialist assessment was associated with high admission rates and the presence of on-site psychiatric departments and DSH teams.}

\section{Conclusions Young DSH patients at risk often go unidentified; as a result their psychological problems may not be treated. Hospitals are frequently unaware of the proportion of patients discharged without adequate assessment.}

Declaration of interest This work was funded by the Department of Health.
The accident and emergency ( $A \& E$ ) clinic is an important gateway to treatment for young deliberate self-harm (DSH) patients. Psychosocial assessment in A\&E clinics must address both short-term and longerterm risks. In the short term, the clinician must be confident that the patient is not going to commit suicide as soon as he or she is discharged. In the longer term, clinicians must be sensitive not only to the risk of repetition or completion, but also to the coexistence of psychosocial problems that require treatment in their own right. In the 12- to 24-year age group, factors associated with high risk of both suicide and attempted suicide are the following: having a previous history of parasuicide; suffering from mental illness - particularly a major depressive disorder - or having a history of mental illness; engaging in substance misuse; lack of adequate parental support, especially when physical or sexual abuse has taken place (Hawton, 1987; Kerfoot, 1988; Marttunen et al, 1991; D'Attilio et al, 1992; Kotila, 1992; Garland \& Zigler, 1993; Kral \& Sakinofsky, 1994; Diekstra et al, 1995).

A straightforward approach to the assessment of patients in the A\&E context may be simply to focus on the identification of recognised problems within this patient group for which referral to medical or social services is appropriate, irrespective of increased risk of subsequent self-harm. This avoids the need for predicting the future and anchors assessment firmly in the area of observable problems.

\section{METHOD}

The study was conducted in two stages.

\section{Stage one: survey Sample}

The first stage involved a postal questionnaire survey of a one-in-three sample of $A \& E$ departments in England, stratified according to size and region. In each hospital a senior A\&E doctor (usually the consultant) and a senior nurse were asked to complete the questionnaire. A total of 118 hospitals were selected, and at least one questionnaire was returned from 107 of these, giving an overall response rate of $91 \%$, with $75 \%$ of doctors and $81 \%$ of nurses responding.

\section{Questionnaire}

Information of as concrete and factual a nature as possible was elicited on the following: the management of DSH patients aged 12-24; any guidelines or policies in operation; the existence of self-harm specialist teams; procedures for assessment, admission and referral of DSH patients; specific training for A\&E staff in the treatment of DSH; and the extent of the statistics collected for auditing and monitoring the levels of service achieved.

\section{Stage two: case study Sample}

In the second stage, $18 \mathrm{~A} \& \mathrm{E}$ departments of various sizes were selected. Hospitals were selected from four of the eight regions in England on the basis of whether they had guidelines and whether a DSH team was operating at the hospital.

\section{Interview}

On average, five members of staff, including a senior doctor, a junior doctor, a nurse and specialist staff, were interviewed in each hospital, using a semi-structured interview schedule. The interview covered their knowledge of hospital policy and guidelines in the area of interest and their observations of how these work in practice, any training they have received in the management of the target group and the factors that influence their assessment and referral practices.

\section{Case notes}

The most recent 50 sets of case notes were examined at each hospital for the target patient group - patients aged 12-24 years who had taken an overdose or injured themselves deliberately (i.e. where notes indicated a deliberate injury). This generated a sample of 894 patients (six representing cases were omitted from the original 900). 


\section{RESULTS}

\section{Psychosocial assessment in} accident and emergency clinics

Eighty-four percent of the 107 hospitals surveyed reported that 12 - to 24 -year-olds were usually given psychosocial assessment in A\&E. However, specialist assessment was only reported to be standard procedure in $44 \%$ of hospitals.

In about two-thirds of cases the specialist available for assessment in $\mathrm{A} \& \mathrm{E}$ was a psychiatrist. Although $45 \%$ of hospitals possessed attached social workers, threefifths of such hospitals reported that social workers rarely or never made psychosocial assessments. This goes some way towards explaining why only about $10 \%$ of hospitals overall reported social workers as playing a major role in this area. Registered mental nurses (RMNs) were even less frequently used (in around $5 \%$ of hospitals).

In the case note sample $(n=894)$, $42.5 \%$ of the patients $(n=380)$ were actually seen by a specialist (either in A\&E or on a ward) $-54 \%$ of 12 - to 15 -year-olds (96 out of 177 cases) and $40 \%$ of 16 - to 24-year-olds (286 out of 717 cases). The questionnaires gave an over-optimistic view of the frequency with which specialist assessment occurred. For example, in those case study hospitals where, according to the questionnaire, patients were always or in most cases seen by a specialist in A\&E, only $26 \%$ were assessed there by a specialist. This tendency to overestimate the frequency with which young DSH patients received specialist assessment was also evident in the interviews.

\section{Admission and specialist assessment on the ward}

Admission significantly increased the likelihood of receiving assessment by a specialist. In the case note study, $67 \%$ of admitted patients $(n=361)$ were seen by a specialist as opposed to only $34 \%$ of patients discharged from A\&E $(n=322) \quad\left(\chi^{2}=75.4\right.$, d.f. $=1, P<0.0001$ ) (self-discharging patients and those receiving psychiatric care at the time were excluded in this comparison as their inclusion could have been misleading $(n=193))$. For younger patients $(n=177)$, for whom admission rates were high (68\%), psychosocial assessment was more frequently carried out on the ward than in $\mathrm{A} \& \mathrm{E}$, and over half the younger group in the case note sample were assessed by a specialist before discharge. However, in less common $(43 \%)$; as a result, specialist assessment on the ward was also less frequent. For this age group, the availability of psychosocial assessment in A\&E is therefore of greater importance.

\section{Psychosocial assessment and the deliberate self-harm team}

Deliberate self-harm teams have been recommended as a way of improving service provision for this patient group, specifically in the areas of psychosocial assessment, referral and aftercare (Department of Health and Social Security, 1984; Royal College of Psychiatrists, 1994). According to the questionnaires, hospitals with a DSH team $(n=25)$ did not offer more frequent specialist assessment in $\mathrm{A} \& \mathrm{E}$, but these assessments were more often carried out by RMNs and social workers and less often by psychiatrists (Table 1$) \quad\left(\chi^{2}=7.43\right.$, d.f. $=1, P<0.006)$. This difference was quite dramatic for the 16- to 24-year-olds. The fact that the presence of a DSH team had a greater impact on the nature of service provision for older patients is to be expected, because DSH teams rarely cover those aged under 16.

According to the case notes, the presence of a DSH team did have an impact on the availability of psychosocial assessment when $A \& E$, ward and out-patient assessment were all included. In particular, a significantly higher percentage of 16- to 24-year-old patients not receiving psychiatric care at the time $(43 \%$ v. $35 \%$, $\chi^{2}=3.9$, d.f. $=1, P<0.05$ ) received a specialist assessment before discharge in hospitals with a DSH team than in hospitals with no team.

It seems that the presence of a DSH team is related to somewhat increased rates of specialist psychosocial assessment but the older group $(n=717)$, admission was

more dramatically to the way in which this specialist assessment is managed.

\section{Guidelines}

The presence or absence of guidelines made no significant difference to the percentage of patients assessed by a specialist. In the seven case-study hospitals with guidelines to refer all patients, $43 \%$ of patients were seen by a specialist compared with $39 \%$ at the seven hospitals with no guidelines on referral. This finding may be explained by junior doctors' ignorance of the guidelines. In only four of the twelve case-study hospitals with guidelines were the junior doctors interviewed aware of the existence of guidelines.

\section{On-site psychiatric services}

In view of the major role played by psychiatrists in specialist assessment, the availability of their services is likely to have an important impact on the frequency with which young patients receive such assessment. When A\&E staff were interviewed, ease of access to the psychiatric service was frequently mentioned as a critical factor in offering specialist psychosocial assessment. As might be expected, medical staff reported easier access to psychiatric specialists if they were nearby. In the survey, $55 \%(33 / 60)$ of hospitals with on-site psychiatric departments reported that most patients were seen by a specialist, as opposed to $32 \%(13 / 41)$ in hospitals where there was no on-site psychiatry $\left(\chi^{2}=5.2\right.$, d.f. $=1, P<0.02$ ).

Similarly, the case note study showed that patients presenting at hospitals with on-site facilities were significantly more likely to receive a specialist assessment than those attending hospitals without $(53 \% v$. $41 \%, \chi^{2}=13.1$, d.f. $\left.=1, P<0.0003\right)$.

Table I Questionnaire data: specialist psychosocial assessment in accident and emergency clinics, broken down by presence or absence of deliberate self-harm (DSH) team

\begin{tabular}{lccccc}
\hline & \multicolumn{2}{c}{ Age I2-I5 } & & \multicolumn{2}{c}{ Age 16-24 } \\
\cline { 2 - 3 } \cline { 5 - 6 } $\begin{array}{l}\text { Patients always or mostly } \\
\text { assessed by }\end{array}$ & $\begin{array}{c}\text { No DSH } \\
\text { team }\end{array}$ & DSH & & No DSH \\
team & & team & DSH \\
\hline Registered mental nurse & $2 \%(2)$ & $4 \%(I)$ & & $9 \%(7)$ & $28 \%(7)$ \\
Social worker & $7 \%(6)$ & $12 \%(3)$ & & $7 \%(6)$ & $12 \%(3)$ \\
Child psychiatrist/psychiatrist & $30 \%(25)$ & $28 \%(7)$ & & $36 \%(30)$ & $8 \%(2)$ \\
Number of hospitals & 82 & 25 & & 82 & 25 \\
\hline
\end{tabular}

Figures represent percentage (number) of hospitals in each group using each type of professional. 
Table 2 shows that the presence of onsite psychiatric facilities is particularly influential for the frequency of specialist assessment for 16- to 24-year-olds at hospitals with no guidelines and no DSH team. At hospitals with guidelines but no DSH team, ease of access is still significant. For hospitals with a DSH team, however, ease of access to the psychiatric department is not a significant factor in providing specialist assessment. This is consistent with the finding that hospitals with DSH teams made greater use of RMNs and social workers and relied less on psychiatrists for specialist assessment.

\section{Quality of non-specialist psychosocial assessment}

Despite the fact that less than half of the young people who present with DSH see a specialist, the survey indicated that the large majority (over $80 \%$ ) will be given a non-specialist, psychosocial assessment in A\&E. The Royal College of Psychiatrists (1994) sanctions such assessments in certain circumstances. The key issue is their adequacy. One guide to the quality of the assessment is the range of information re corded in the case notes by the A\&E staff. Table 3 compares the frequency with which specialists and non-specialists record key aspects of psychosocial assessment in patients' case notes. Because patients who are admitted may not be conscious while in $\mathrm{A} \& \mathrm{E}$ or may be expected to receive a fuller assessment by medical staff on the ward, the lack of a full assessment in $\mathrm{A} \& \mathrm{E}$ cannot be regarded as poor practice; such patients have therefore been omitted from the table, as have self-discharging patients and those in psychiatric care at the time. The case notes of patients seen by specialists in A\&E were significantly more likely to contain key information (psychiatric history, mental state, social situation, precipitating event, severity of intent and risk of repetition) than those of patients assessed by a non-specialist.

In addition, the staff interviewed in the 18 case-study hospitals were asked about the adequacy of psychosocial assessment made by non-specialist doctors in A\&E. In 10 of the 18 hospitals, senior medical staff reported that the junior doctors usually responsible were ill-equipped to make such assessments adequately, owing to lack of time, lack of experience or lack of concern with the psychological aspects of treatment. In only two of these hospitals did senior
Table 2 Case note data: percentage of patients assessed by specialist broken down by presence or absence of on-site psychiatric services and by presence or absence of guidelines and deliberate self-harm (DSH) team

\begin{tabular}{|c|c|c|c|}
\hline & \multicolumn{2}{|c|}{$\begin{array}{l}\text { Percentage (number) of patients receiving } \\
\text { specialist assessment }\end{array}$} & \multirow[b]{2}{*}{$\begin{array}{l}\text { Statistical } \\
\text { significance }\end{array}$} \\
\hline & $\begin{array}{c}\text { Hospitals with } \\
\text { psychiatric facilities } \\
\text { 'on-site' }\end{array}$ & $\begin{array}{c}\text { Hospitals with } \\
\text { psychiatric facilities } \\
\text { detached }\end{array}$ & \\
\hline All patients & $53 \%(447)$ & $41 \%(447)$ & $\chi^{2}=13.09, P<0.0003$ \\
\hline Patients aged $12-15$ & $67 \%(93)$ & $61 \%(84)$ & NS \\
\hline Patients aged 16-24 & $50 \%(354)$ & $37 \%(363)$ & $\chi^{2}=12.5, P<0.0004$ \\
\hline \multicolumn{4}{|l|}{ Patients aged $16-24$} \\
\hline \multicolumn{4}{|l|}{ Hospitals with no } \\
\hline guidelines/no DSH team & $61 \%(70)$ & $24 \%(I 12)$ & $\chi^{2}=25.35, P<0.0001$ \\
\hline \multicolumn{4}{|l|}{ Hospitals with } \\
\hline guidelines/no DSH team & $48 \%(163)$ & $32 \%(82)$ & $\chi^{2}=6.26, P<0.02$ \\
\hline Hospitals with DSH team & $45 \%(12 I)$ & $47 \%(169)$ & NS \\
\hline
\end{tabular}

doctors consider that assessments made by junior doctors were generally adequate or good. The senior doctors in the other 16 hospitals felt that quality of assessment by junior doctors varied with the quality of the senior house officers (SHOs), their experience and the pressures of the time required to undertake a thorough assessment. In those hospitals where there was concern over the quality of non-specialist assessment, senior clinicians frequently reported that admission and specialist assessment were the rule and that this compensated for the problems associated with the non-specialist. However, the case notes demonstrated that many patients were not receiving this specialist attention.

Three-quarters of the SHOs interviewed reported that when they carried out a psychosocial assessment they always attempted to establish a psychiatric history and to assess the risk of further self-harm and the intent of the patient. Just over a half said that they did a mental state examination. However, the focus was on short-term risk - in 11 of the 18 hospitals there was unequivocal evidence from the interviews that the psychosocial assessments carried out in $\mathrm{A} \& \mathrm{E}$ clinics were centrally concerned with the short-term risk of repeated attempts or suicide. Short-term risk was also cited as the deciding factor in immediate referral to a specialist.

\section{Selection for specialist assessment}

Some researchers have argued that the psychosocial prognosis for a proportion of DSH presentations is good and that treatment may therefore be unnecessary
Table 3 Case note data: information recorded in accident and emergency clinics by specialists and by nonspecialists (excluding patients in psychiatric care at the time, patients self-discharging and patients admitted)

\begin{tabular}{lccc}
\hline $\begin{array}{l}\text { Case notes include } \\
\text { assessment of }\end{array}$ & $\begin{array}{c}\text { Not assessed by } \\
\text { specialist }(\%(n))\end{array}$ & $\begin{array}{c}\text { Assessed by specialist } \\
(\%(n))\end{array}$ & $\begin{array}{c}\text { Statistical } \\
\text { significance }\end{array}$ \\
\hline Psychiatric history & $42 \%(94)$ & $69 \%(63)$ & $\chi^{2}=19.13, P=0.0000$ I \\
Mental state & $41 \%(94)$ & $73 \%(66)$ & $\chi^{2}=26.61, P=0.0000$ I \\
Social situation & $33 \%(75)$ & $71 \%(65)$ & $\chi^{2}=37.37, P=0.0000$ I \\
Precipitating event & $65 \%(143)$ & $79 \%(73)$ & $\chi^{2}=6.93, P=0.008$ \\
Estimate of severity of intent & $50 \%(112)$ & $77 \%(70)$ & $\chi^{2}=19.37, P=0.00001$ \\
Risk of repeated attempt & $47 \%(106)$ & $67 \%(60)$ & $\chi^{2}=10.53, P=0.001$ \\
Total number of case notes & 228 & 90 & \\
\hline
\end{tabular}


(Hawton, 1987; Hurry \& Storey, 1999). This may mean that the level of specialist assessment identified in the present study is fairly adequate. However, such a conclusion would rely on the assumption that those selected for specialist assessment are the 'right' ones. Table 4 presents the relationship between key patient characteristics and whether 16- to 24-year-olds are assessed by a specialist. A series of logistic regressions was carried out in order to establish statistically significant effects.

Consistent with the interviews, where notes recorded patients either as having suicidal intent or as being at risk of subsequent attempts, they were much more likely to see a specialist. Patients with past psychiatric care or those diagnosed with depression were also more likely to be passed to a specialist, but it is likely that the presence or absence of depression was frequently not established, and only a little over half of those diagnosed with depression were actually seen by a specialist. Patients with a previous recorded episode of self-harm were not significantly more likely to see a specialist. Only half the patients who had made a previous attempt were assessed by a specialist. Where alcohol was involved, patients were no more likely to see a specialist; where drugs were involved, they were significantly less likely to be seen. We failed to find any evidence that guidelines or the existence of a DSH team improved the selection process. The existence of a DSH team did increase the likelihood of specialist assessment but it did so equally for patients with no risk and those with some risk.

\section{DISCUSSION}

\section{The current position}

When young people present at A\&E clinics following DSH they are likely to receive thorough treatment of their medical problems. The psychological cause of their injury requires an equally painstaking assessment, yet it is estimated that nationally only $54 \%$ of 12 - to 15 -year-olds and $40 \%$ of $16-$ to $24-$ year-olds are assessed by a specialist. A recently published report (Kapur et al, 1998) drawing data from four teaching hospitals not included in our study found a similar percentage $(46 \%)$ of adult self-harm patients discharged without specialist assessment.

Table 4 Case study data: patients aged 16-24 receiving specialist assessment before or after discharge broken down by patient presentation characteristics (excludes patients already receiving psychiatric care)

\begin{tabular}{lrllc}
\hline & $n$ & $\begin{array}{c}\text { Percentage } \\
\text { assessed }\end{array}$ & $\begin{array}{c}\text { Odds } \\
\text { ratio }\end{array}$ & $\begin{array}{c}\text { Logistic regression - } \\
\text { Wald analysis }\end{array}$ \\
\hline Residence & & & & \\
$\quad$ With patients/carers & 185 & $50 \% * *$ & 1.62 & - \\
$\quad$ In institution & 43 & $43 \%$ & - & 7.09 \\
As lone parent & 12 & $92 \%^{* *}$ & 16.06 & 4.04 \\
$\quad$ Alone/no fixed abode & 49 & $55 \% *$ & 1.83 & - \\
$\quad$ With partner & 64 & $41 \%$ & - & - \\
Previous deliberate self-harm & 199 & $50 \%$ & - & 7.69 \\
Past psychiatric care & 64 & $58 \%^{* *}$ & 2.10 & 2.18 \\
Notes record patient depressed & 163 & $50 \% * *$ & 1.63 & - \\
Street drugs involved & 76 & $16 \% * * *$ & 0.23 & 18.04 \\
Alcohol involved & 234 & $38 \%$ & - & 12.55 \\
Suicide risk & 68 & $76 \% * * *$ & 3.98 & 2.44 \\
Suicidal intent & 132 & $64 \% * * *$ & & \\
\hline
\end{tabular}

$* P<0.05, * * P<0.01, * * * P<0.001$.

\section{Non-specialist assessment}

Although less than half of young self-harm patients receive a specialist assessment, most are assessed by an A\&E doctor. However, the evidence from the present study suggests that such assessments are likely to be inadequate. In the majority of case notes reviewed, important information concerning previous psychiatric history, current mental state and even risk of a repeated attempt was not recorded if the patient had not been seen by a specialist. Similar levels of important omissions in the case notes of self-harm patients have been found by others (O'Dwyer et al, 1991; Ebbage et al, 1994). It is possible that these areas were covered but simply not recorded. However, junior doctors almost invariably claimed to record all information elicited on risk, present mental state and history of psychiatric care or previous attempts. Also, the overwhelming majority of staff interviewed in the 18 case-study hospitals felt that junior doctors were unreliable as psychosocial assessors, particularly in the area of long-term risk.

\section{Assessment in the $A \& E$ context}

The A\&E clinic is geared towards the immediate, and towards the physical rather than the psychological. Junior doctors working in such an environment are not encouraged to spend with patients the time that psychosocial assessment requires. The underlying problems of depression, family upheavals and alcohol and drug misuse, which may be at the root of self-harm, require treatment, but in the short-term view of the $A \& E$ doctor they will tend to be ignored in favour of risk factors surrounding the likelihood of immediate repetition. It is questionable whether an $\mathrm{A} \& \mathrm{E}$ junior doctor can reliably determine something as complex as suicidal intent in a young adolescent, or whether A\&E where the young person may be suffering from the effects of an overdose and where parents may be emotional, angry or feeling guilty - is the best place to make such an assessment.

\section{Specialist assessment}

Patients with certain risk factors, such as depression or a previous history of psychiatric care, recorded in their case notes, were more likely to be assessed by a specialist, but even in this group rates of discharge without specialist assessment were worryingly high. Despite the implications for risk of both re-attempting and other negative sequelae, 16- to 24-year-olds whose selfharm attempt reportedly involved alcohol were not typically seen by a specialist, while those using street drugs were significantly less likely to receive specialist attention than other self-harm patients. The percentage of patients with a clear indication of these risk factors not being seen by a specialist is a cause for concern. 


\section{Understanding levels of service provision}

Senior clinicians want to provide a good service and are aware of the problems of non-specialist assessment in A\&E. However, they tend to overestimate both the proportion of young self-harm patients admitted and the proportion assessed by a specialist. Where short-term risk is the key to immediate specialist referral, patients with chronic difficulties but no serious suicidal intent will receive little more than a medical response to their crisis. The available evidence suggests that, in the opinion of clinicians, the large majority of young self-harm patients do not have serious suicidal intent (Hawton et al, 1982a,b; Hurry \& Storey, 1999).

Psychiatrists are normally responsible for specialist assessment, but many selfharm patients may not be diagnosed at clinical interview as suffering from the classic mental illnesses such as depression, anxiety or schizophrenia - perhaps only about a quarter or a third are so diagnosed (Hawton, 1986; Kerfoot, 1988; House et al, 1992). The psychological problems associated with repeated self-harm, with alcohol and drug misuse and with a generally chaotic lifestyle are less comfortably embraced by psychiatry. This may explain the fact that young self-harm patients involved with alcohol and drug misuse do not attract particular attention despite the fact that they are in a high-risk group for attempting, for suicide and for other negative psychosocial sequelae.

\section{Future implications}

An important gateway to treatment for young people who deliberately harm themselves is appropriate psychosocial assessment following hospital presentation. Such assessment is only reliably given by a specialist. The factors associated with increased rates of specialist assessment are high in-patient admission rates, the presence of a DSH team and the presence of on-site psychiatric facilities. The evidence presented cannot demonstrate a causal relationship between in-patient admission, DSH teams, on-site psychiatry and increased rates of specialist assessment. However, the range of potential options identified offers hospitals a useful degree of flexibility when planning their response to this patient group. The case-study hospitals not adopting one of these strategies had

\section{CLINICAL IMPLICATIONS}

Closer monitoring of levels of specialist assessment of deliberate self-harm (DSH) patients presenting at accident and emergency (A\&E) clinics would be desirable.

- We recommend the introduction of measures to facilitate specialist assessment, such as high rates of admission, a DSH specialist in A\&E or good working relations between $A \& E$ and on-site psychiatric departments.

Assessment should focus on identification of significant psychosocial problems in $A \& E$, not just short-term risk of suicide or repetition.

\section{LIMITATIONS}

We relied on case notes for information on patient characteristics.

- The study lacked detailed data on the process of specialist assessment.

- There was no measure of patient outcomes in order to evaluate the effect of specialist assessment.

JANE HURRY, PhD, Child Development and Learning, Institute of Education, London; PAMELA STOREY, PhD, Thomas Coram Research Unit, Institute of Education, London.

Correspondence: Dr J. Hurry, Institute of Education, University of London, 20 Bedford Way, London WCIHOAL

(First received 9 March 1999, final revision 6 July 1999, accepted 9 July 1999)

very low rates of specialist assessment (Hurry \& Storey, 1999).

The relatively low rates of specialist assessment offered to young self-harm patients, even those at quite high risk of long-term problems, should cause both $\mathrm{A} \& \mathrm{E}$ and psychiatric departments to reassess their provision. An important finding emerging from our study was the lack of awareness of senior clinicians of the level of service provided to young self-harm patients. Audit of service provision may be the first step necessary at the local level to identify difficulties.

There is a particular need to address the specific and problematic nature of this patient group. House et al (1992) commented that there is "a general perception that psychiatric intervention is ineffective for patients who are admitted to hospital having harmed themselves", and they remark that this in itself does not encourage $\mathrm{A} \& \mathrm{E}$ departments to take psychosocial assessment and referral very seriously. We need to know more about how to treat these patients effectively; this should be the focus of future research.

\section{ACKNOWLEDGEMENTS}

We thank the many doctors and nurses who gave their time so generously.

\section{REFERENCES}

D’Attilio, J., Campbell, B., Lubold, P., et al (1992) Social support and suicide potential: preliminary findings for adolescent populations. Psychological Reports 70, 76-78

Department of Health and Social Security (1984) The Management of Deliberate Self-harm, HN(84)25. London: Department of Health and Social Security.

Diekstra, R., Keinhorst, C. \& de Wilde, E. (1995) Suicide and suicidal behaviour among adolescents. In Psychosocial Disorders in Young People: Time Trends and Their Causes (eds M. Rutter \& D. Smith). Chichester: John Wiley \& Sons.

Ebbage, J., Farr, C., Skinner, D. V., et al (1994) The psycho-social assessment of patients discharged from accident and emergency departments after deliberate self-poisoning. Journal of the Royal Society of Medicine, 87, $515-516$

Garland, A. \& Zigler, E. (1993) Adolescent suicide prevention: current research and social policy implications. American Psychologist, 48, 169-182.

Hawton, K. (1986) Suicide and Attempted Suicide among Children and Adolescents. London: Sage. 
— (1987) Assessment of suicide risk. British Journal of Psychiatry, 150, 145-153.

_, Cole, D., O'Grady, J., et al (1982a) Motivationa aspects of deliberate self-posioning in adolescents. British Journal of Psychiatry, 14I, 286-291.

—, Osborn, M., O'Grady, J., et al (1982b)

Classification of adolescents who take overdoses. British Journal of Psychiatry, 140, 124-131.

House, A., Owens, D. \& Storer, D. (1992)

Psycho-social intervention following attempted suicide: is there a case for better services? International Review of Psychiatry, 4, 15-22.
Hurry, J. \& Storey, P. (1999) Deliberate Self-harm in Young People: The Hospital Response. London: Department of Health.

Kapur, N., House, A., Creed, F., et al (1998) Management of deliberate self poisoning in adults in four teaching hospitals: a descriptive study. British Medical Journal, 316, 831-832.

Kerfoot, M. (1988) Deliberate self-poisoning in childhood and early adolescence. Journal of Child Psychology and Psychiatry, 29, 335-344.

Kotila, L. (1992) The outcome of attempted suicide in adolescence. Journal of Adolescent Health, 13, 4I5-4I7.

Kral, M. \& Sakinofsky, I. (1994) Clinical model for suicide risk assessment. Death Studies, 18, 31।-326.
Marttunen, M. J., Aro, H. M., Henriksson, M. M., et al (1991) Mental disorder in adolescent suicide. DSMIII-R Axes I and II among 13 to 19 year olds. Archives of General Psychiatry, 48, 834-839.

O'Dwyer, F., D'Alton, A. \& Pearce, J. B. (199I)

Adolescent self-harm patients: audit of assessment in an accident and emergency department. British Medical journal, 303, 629-630.

Royal College of Psychiatrists (1994) The General Hospital Management of Adult Deliberate Self-Harm. A Consensus Statement on Standards for Service Provision. Council Report CR32. London: Royal College of Psychiatrists. 